\title{
PROPOSTA DE NIVELAMENTO EM BIM PARA OS DISCENTES E EGRESSOS DO CURSO DE ENGENHARIA CIVIL DA UNIVERSIDADE FEDERAL DO CEARÁ
}

\author{
DOI: 10.37702/2175-957X.COBENGE.2021.3670
}

LUCIANO HAMED CHAVES HAIDAR SOUSA - lucianohamed@alu.ufc.br UNIVERSIDADE FEDERAL DO CEARÁ AVENIDA SANTOS DUMONT 3665

60150-162 - FORTALEZA - CE

SABRINA RODRIGUES GONCALVES - sabrinarg57@gmail.com

Universidade Federal do Ceará

Rafael Dantas 2236

60830-585 - Fortaleza - CE

CELY MARTINS SANTOS DE ALENCAR - celyms@gmail.com

UFC

Campus do Pici, Bl710 s/n

60455-900 - FORTALEZA - CE

Resumo: Na esfera do curso de Engenharia Civil da Universidade Federal do Ceará, existe uma carência no que tange as aplicações do Building Information Modeling BIM. Diante disso, foram necessárias ações complementares permeando a graduação, como a criação de uma disciplina optativa, realização de eventos, bem como a criação de um projeto de extensão. Nesse cenário, até então as ações desenvolvidas de forma mais intensiva foram direcionadas aos discentes do curso, como é o caso da disciplina optativa e do projeto de extensão supracitado. As Diretrizes Curriculares Nacionais do curso de engenharia civil, recomenda o desenvolvimento de atividades que aproximem os discentes do ambiente profissional. Nesse contexto, temos a estratégia BIM BR que dentre seus objetivos, traz o estimulo a capacitação em BIM, dentre outros. Assim, objetiva-se com este trabalho, propor um nivelamento do BIM, associado a ferramentas e tecnologias complementares, aos discentes e egressos do curso de Engenharia Civil, com o fito de fomentar o BIM integrado entre a academia o mercado de forma mais incisiva e direcionada. Nesse cenário, serão desenvolvidos quatro módulos de capacitação sob temáticas: BIM, Lean, Programação visual, realidade virtual e aumentada. 


\section{COBENGE de Educação em Engenharia

Esses módulos serão conduzidos de forma colaborativa entre os discentes, egressos e profissionais convidados, a fim de promover o engajamento dos envolvidos. Espera-se que ao final do processo, os participantes desenvolvam tanto relatos de experiências para que outras universidades possam replicar a ação, como ainda, desenvolvam novas soluções no tocante a engenharia do que foi experienciado.

Palavras-chave: BIM, Engenharia Civil, UFC, nivelamento 


\section{PROPOSTA DE NIVELAMENTO EM BIM PARA OS DISCENTES E EGRESSOS DO CURSO DE ENGENHARIA CIVIL DA UNIVERSIDADE FEDERAL DO CEARÁ}

\section{INTRODUÇÃO}

No contexto do curso de Engenharia Civil da Universidade Federal do Ceará (UFC), tem-se uma grade curricular que foi modelada em outubro de 2004, segundo o Projeto Político Pedagógico do curso. Essa grade, além de não contemplar os avanços tecnológicos que tivemos na última década, apresenta a problemática de que os conteúdos não são interligados entre si, salvo poucas exceções.

No âmbito dos avanços tecnológicos na área das engenharias e de encontro com a multidisciplinariedade, tem-se a tecnologia Building Information Modeling - BIM, definida por Kymell (2008), como a simulação de um projeto, que consiste na modelagem 3D dos componentes do mesmo, agregando-se toda a informação requerida ao seu planejamento, à construção ou operação, e ao encerramento do ciclo de vida da edificação. No curso de engenharia civil da UFC, existe uma carência no que tange as aplicações do Building Information Modeling - BIM. Segundo Gonçalves et al. (2020) apesar de o conceito de BIM e percepção de sua relevância estarem melhor difundidos no curso em comparação com anos anteriores, o BIM não se encontra consolidado de forma integrada e consistente, tendo-se apenas aplicações pontuais de ferramentas BIM em algumas disciplinas, como Desenho para Engenharia, Gerenciamento para Construção Civil e Projeto e construção de edifícios.

Dessa forma, a formação dos egressos deixa lacunas no tocante ao BIM, suas tecnologias e metodologias associadas. Isso, gera disparidades entre o que é esperado dos recém formados no mercado e o que a universidade consegue entrega-los. Além disso, quando a academia consegue apresentar ao discente conteúdos aliados a prática e inovações tecnológicas consegue despertar a motivação dos mesmos, contribuindo consequentemente para menores evasões no curso, conforme mostrado por Corrêa (2019) que defende que a aprendizagem de BIM exerce papel na motivação dos estudantes e por Lima (2020 apud FERRANDIZ et al, 2017) que comprova que a ferramenta BIM pode reduzir problemas relacionados ao desempenho do aluno, além de aumentar sua motivação e satisfação.

Dessa forma, o objetivo deste trabalho é apresentar uma proposta de nivelamento em BIM no curso de engenharia civil da Universidade Federal do Ceará, com o intuito de reparar as lacunas existentes na formação dos egressos e dos discentes, com um plano de capacitação em BIM e suas metodologias associadas.

\subsection{Building Information Modelling}

Para Villagrasa (2021) BIM é uma ferramenta de trabalho, uma metodologia multidisciplinar. Para Azhar et al (2012) o processo BIM envolve disciplinas e sistemas em um único modelo virtual, corroborando com a integração e colaboração entre os diferentes agentes envolvidos no projeto.

Pereira e Ribeiro (2015) defendem a necessidade de uma estratégia pedagógica que possa melhor integrar os conhecimentos da aplicação da ferramenta BIM com as necessidades do ensino de Desenho e Projeto na Engenharia Civil em todos os âmbitos: Arquitetônicos, Estruturais, de Infraestrutura, dentre outros. Essa necessidade é ainda mais evidenciada no contexto brasileiro, onde a Estratégia BIM BR instituída em 2018 e 
atualizada em agosto de 2019 pelo decreto $N^{\circ} 9.983$ preconiza a disseminação do BIM no país, estimulando a maior inserção do BIM nas disciplinas de graduação e pós-graduação em Engenharia e Arquitetura e o desenvolvimento e a aplicação de novas tecnologias relacionadas ao BIM.

Ainda no âmbito da legalidade do BIM, seu uso obrigatório em obras públicas é assegurado pelo Decreto no 10.306, de 2 de abril de 2020 que estabelece a utilização do Building Information Modeling na execução direta ou indireta de obras e serviços de engenharia realizada pelos órgãos e pelas entidades da administração pública federal. Nesse sentido, tem-se, ainda, conforme a Lei de Licitações e Contratos Administrativos, a Lei no 14.133, de 1 de abril de 2021:

\begin{abstract}
Nas licitações de obras e serviços de engenharia e arquitetura, sempre que adequada ao objeto da licitação, será preferencialmente adotada a Modelagem da Informação da Construção (Building Information Modeling$B I M)$ ou tecnologias e processos integrados similares ou mais avançados que venham a substituí-la.
\end{abstract}

Dessa maneira, o BIM, deve ser preferencialmente utilizado nos órgãos públicos dos âmbitos, federal, estadual e municipal. Para isso, é urgente que a geração de engenheiros em formação encontre, ainda na universidade, um ambiente de estímulo ao uso e desenvolvimento do BIM, onde estes possam entrar em contato com as tecnologias necessárias e sejam capazes desenvolver os conhecimentos, as habilidades e as atitudes consideradas necessárias para se trabalhar com o paradigma BIM, dentre estas o desenvolvimento de soluções criativas e inovadoras, a colaboração, o trabalho em equipe e a liderança e a habilidade de desenvolver uma comunicação efetiva (CHECCUCI, 2014).

\title{
1.2 A programação visual
}

Para Coco e Celani (2021) o BIM habilita a construção civil para a era da Informação, permitindo o engate de diversas tecnologias e modelos de negócio associadas à indústria 4.0. Nesse sentido, a programação visual se torna uma ferramenta poderosa aliada ao BIM. A linguagem de programação visual, é composta de elementos pictóricos que internamente possuem um código textual para executar algum tipo de ação, a partir dos dados de entrada. Segundo o BIM Dictionary (2019), ao contrário de uma linguagem textual (por exemplo, Python ou C \#), uma Linguagem de Programação Visual permite aos usuários codificar e manipular (projetar) regras usando uma série de elementos gráficos que representam entidades e suas relações. Estas regras, ao serem processadas em aplicações semelhantes a Dynamo ${ }^{\circledR}$, Grasshopper ${ }^{\circledR}$ e Generative Components $\AA$, irão gerar ou modificar um Modelo Gerativo, um Modelo Paramétrico ou seus Componentes de Modelo.

Pela interface mais intuitiva e a facilidade de execução de ações com poucos passos, a programação visual se torna mais promissora devido a curva de aprendizado em relação a programação textual ser menor. Diante disso, o uso da programação visual se torna uma ferramenta bastante relevante associada ao BIM para a resolução de problemas de engenharia.

\subsection{Lean Construction}

A construção enxuta (Lean Construction), surgiu como uma adaptação da produção enxuta e do sistema Toyota de produção. Nessa perspectiva, temos como precursor desta filosofia nesse campo Koskela et al. (2002), que afirma que a produção enxuta exige três ações possíveis, sendo elas: desenho do sistema de produção, controle do sistema de 
produção e melhoria do sistema de produção. Apud (LIMA e RIBEIRO, 2020). Dessa forma, temos o Lean como uma abordagem de construção de sistemas de produção para gerar o máximo de valor possível nas atividades de engenharia, diminuindo desperdícios de materiais, de esforço e de tempo sempre alinhado com o que os clientes necessitam.

Para Borges et al (2018) a problemática que o Lean propõe dirimir, no tocante ao auxílio na eficiência dos processos construtivos, o BIM permite a execução de atividades com mais eficiência, transparência entre as partes interessadas.

Para Eastman (2014), BIM e Lean são evoluirão em conjunto, devido aos seus conceitos serem complementares. Quando aplicado ao processo projetivo o Lean traz resultância na redução de desperdícios através da eliminação de etapas que não geram valor para o cliente. Traz ainda o conceito de projeto simultâneo para eliminar erros e retrabalhos gerando mais eficiência. $E$ ainda, possibilita a redução da duração do ciclo, e o BIM viabiliza os conceitos supracitados.

Ademais, o Lean pode ser associado a outras metodologias ágeis de gestão, gerando um método customizado a cada realidade de empresa. Para a viabilidade, podese citar o kanban que funciona como uma grande lousa com informações que auxiliam na gestão visual, e, ainda, o Scrum, que segundo Goes (2019) é estruturado em eventos, papéis e artefatos.

\section{$1.4 \quad$ Realidade virtual e aumentada}

A Realidade Virtual (RV) é visualizada como a forma mais avançada de interface de usuário de computador disponível atualmente. Através dela, o indivíduo é capaz de vivenciar mundos inexistentes fisicamente, por meio de equipamentos que transmitem a nítida impressão de estar no ambiente gerado em computador, proporcionando uma "interação natural" com os ambientes sintéticos por ele gerado. (SILVA et al ,2009).

A tecnologia RV possui inúmeras aplicações, em especial na construção civil, e a tendência é crescer intensivamente nos escritórios e imobiliárias. Seu uso se expande juntamente com a ampliação da metodologia BIM levando qualidade e satisfação para aqueles que a utilizam em conjunto. (SILVA et al ,2009).

Para Lucena (2019), por meio da realidade virtual e da dinâmica dos jogos, é possível atribuir um papel mais ativo ao usuário em seu próprio processo de aprendizado, de modo a exercitar suas capacidades para tomada de decisões competentes.

\subsection{O Escritório de Projetos Integrados de Engenharia - EPE}

O EPE é um projeto de extensão da Universidade Federal do Ceará criado em 2017 no Departamento de Integração Acadêmica e Tecnológica - DIATEC da universidade. Desde então, o projeto realiza ações com o propósito de fomentar a filosofia BIM no meio acadêmico e ser o elo entre o mercado e a academia. Entre as atividades que o projeto desenvolve atualmente destaca-se o apoio e o suporte à inclusão do BIM em disciplinas do curso de engenharia civil como Desenho para Engenharia, Gerenciamento para Construção Civil e Modelagem da Informação e da Construção.

\section{METODOLOGIA}

A metodologia deste trabalho consistiu em uma revisão bibliográfica e uma pesquisa de campo realizada através de questionários eletrônicos e enviados aos alunos do curso de engenharia civil da Universidade Federal do Ceará do Campus do Pici com o intuito de conhecer a percepção dos discentes sobre o tema e traçar a proposta com base nos 
interesses dos discentes. $\mathrm{O}$ esquema de delineamento da pesquisa é mostrado na figura 1:

Figura 1: Metodologia da pesquisa

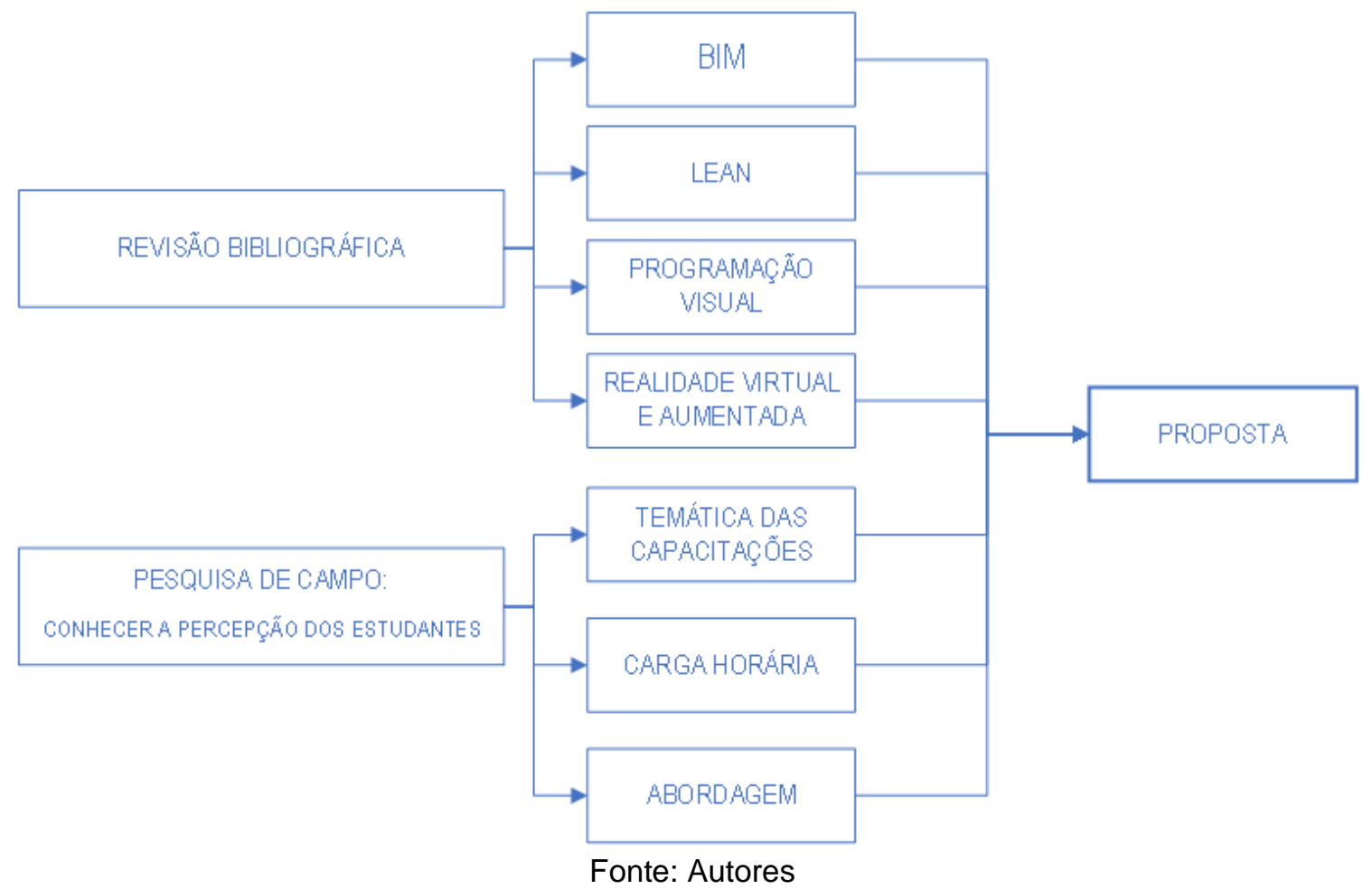

\section{DISCUSSÃO DOS RESULTADOS E PROPOSTA}

\subsection{A pesquisa}

A pesquisa teve a participação de discentes do curso de engenharia civil da UFC $(80 \%)$ e egressos, de forma que a proposta de nivelamento elaborada contempla os dois perfis de estudantes, promovendo, ainda, maior integração entre eles. (Figura 2)

Figura 2: Situação dos participantes da pesquisa

Você se enquadra em qual das opções abaixo?

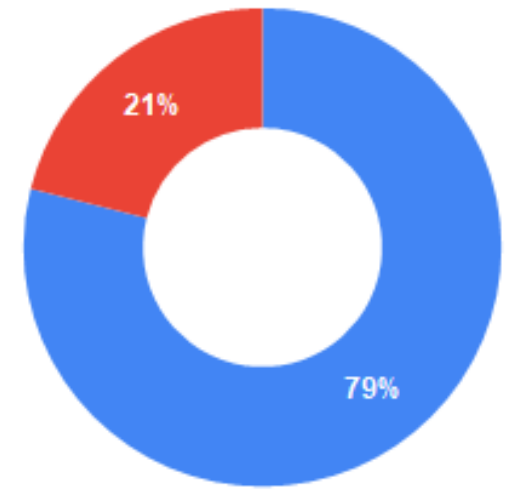

- Discente (Sou aluno do curso)

- Egresso (Concluí o curso)

Fonte: Dados da pesquisa 
Os alunos foram questionados acerca do aprendizado de BIM e suas tecnologias associadas no decorrer do curso e, quase $80 \%$ dos participantes considera que não aprendeu a temática ao longo de seu período de graduação (Figura 3). Esse resultado manifesta uma lacuna entre o que é mercado demanda e o que os alunos tem contato na graduação.

Figura 3: Percepção do aprendizado de BIM

Você considera que aprendeu BIM e suas tecnologias associadas durante o período do curso?

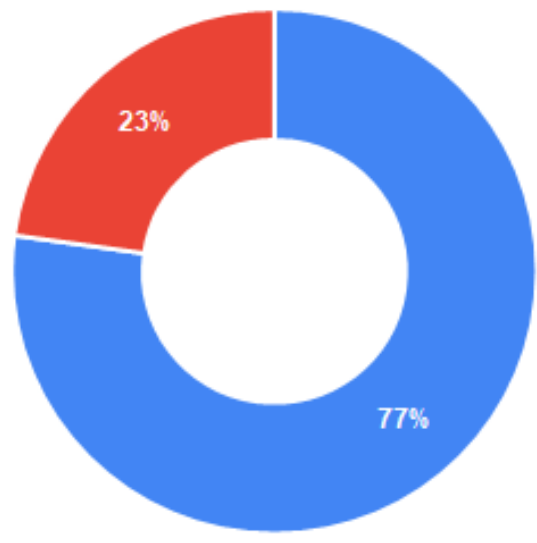

Fonte: Dados da pesquisa

Dentre as temáticas elencadas nesta pesquisa, $40 \%$ dos alunos acreditam que BIM seja a mais relevante para a formação profissional (Figura 4), o que sugere que os discentes têm uma percepção da crescente demanda do mercado pelo uso do BIM.

Figura 4: Temáticas mais relevantes

Qual das temáticas abaixo você considera relevante na sua formação profissional?

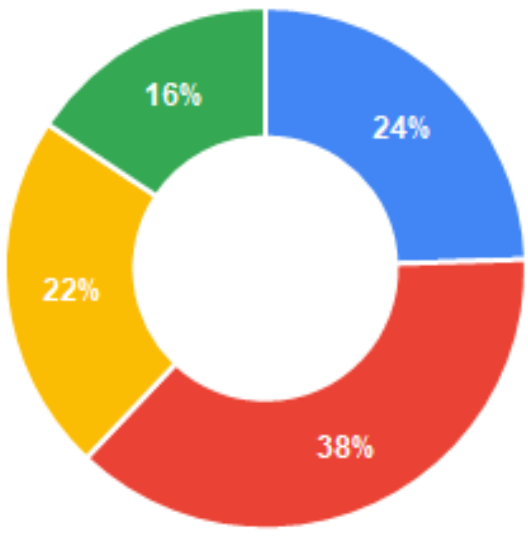

- LEAN CONSTRUCTION

- BIM

" PROGRAMAÇÃO VISUAL

- REALDADE VIRTUAL E AUMENT ADA

Fonte: Dados da pesquisa 
Com o intuito de conhecer a percepção dos estudantes acerca da abordagem dos temas, os estudantes mostraram preferência pela aprendizagem baseada em problemas (APB) e metodologias híbridas entre conteúdos expositivos e trabalhos aplicados. Apenas 9\% dos alunos acreditam que aprendizagem passiva e expositiva seja a melhor abordagem para construção do aprendizado (Figura 5). Esse resultado evidencia a necessidade de uma ruptura com modelos tradicionais de ensino, de modo que o nivelamento foi elaborado de forma a dar uma vivência prática e aproximar os alunos do mercado.

Figura 5: Abordagem dos temas

Como você acredita que deve ser o aprendizado sobre as temáticas citadas anteriormente?

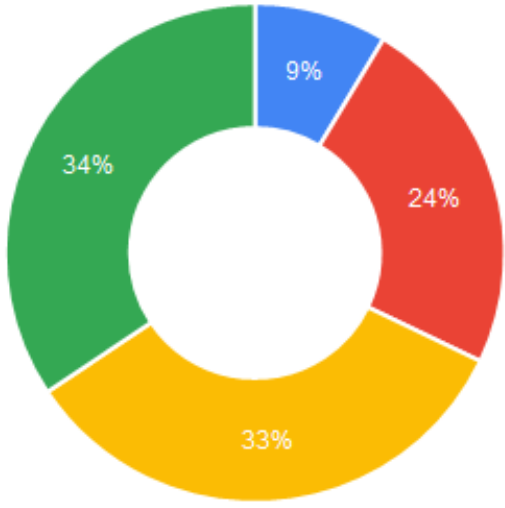

- Aprendizagem passiva expositiva

- Apreendizagem cooperativa

- Aprendizagem baseada em problemas

- Híbrido entre exposição de conteúdos e trabalhos aplicados.

Fonte: Dados da pesquisa

No que diz respeito a carga horária ideal para o nivelamento, as respostas variaram entre 15, 30 e 60 dias, considerando uma hora de dedicação diária. Para essa proposta, sugere-se um tempo médio de 45 dias para cada temática.

Figura 6: Carga horária

Qual a duração que você acredita que deve ser dedicada a cada tema? (Considerando em média 1h por dia)

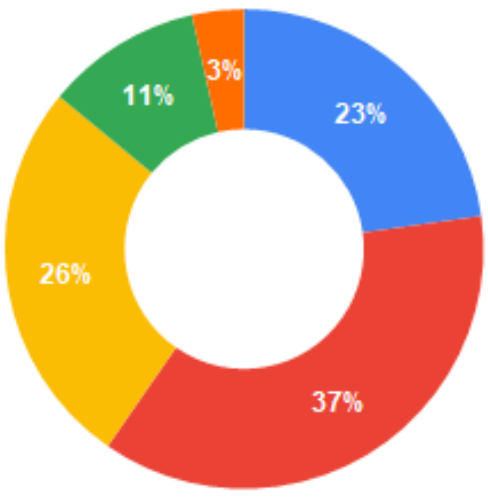




\subsection{Proposta}

O nivelamento será realizado com a duração de 45 dias a cada semestre. Será gerido pelo Escritório de Projetos Integrados de Engenharia - EPE. Acontecerá no Centro de Tecnologia da Universidade Federal do Ceará de forma híbrida, com atividades síncronas e assíncronas. A sequência das macro etapas que serão realizadas durante cada semestre são mostradas na figura7.

Figura 7: Macro etapas de módulos de nivelamento

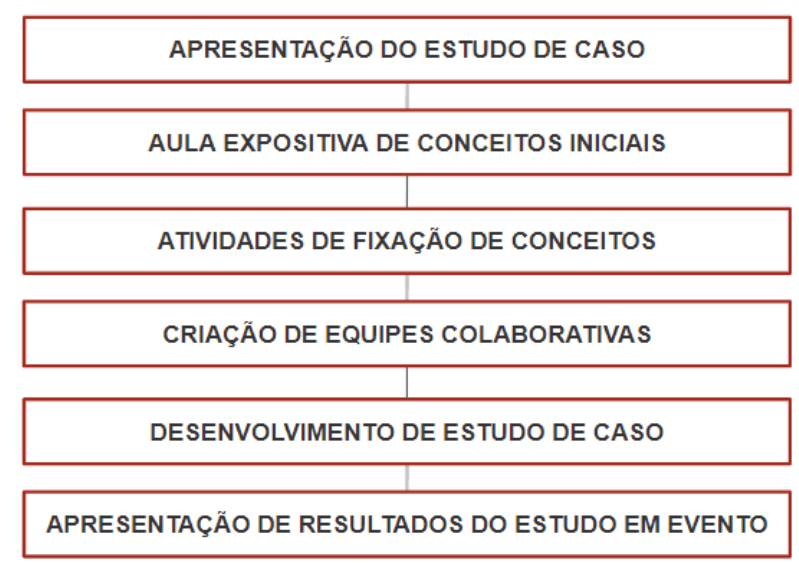

Fonte: Os autores.

Inicialmente a proposta do estudo de caso, que será planejado pelo Escritório de Projetos Integrados de Engenharia, será apresentada aos participantes do nivelamento. Em seguida, serão explorados conceitos básicos por meio de exposição de convidados especialistas no tema, mesclado com o uso de aprendizagem cooperativa para promover engajamento do grupo durante 0 ciclo. Ademais, serão realizados testes rápidos para verificação do entendimento dos participantes sobre o assunto e baseado nos resultados podem ser tomadas medidas de reforço de conteúdo. Posteriormente serão criados grupos mesclando discentes e egressos do curso em engenharia civil, promovendo a integração entre academia e mercado, para o desenvolvimento dos estudos de caso. Cada módulo de nivelamento terá um tema definido. Iniciando pelo de BIM, em seguida Lean, programação visual e realidade virtual e aumentada, de acordo com a figura 8.

Figura 8: Macro etapas de módulos de nivelamento

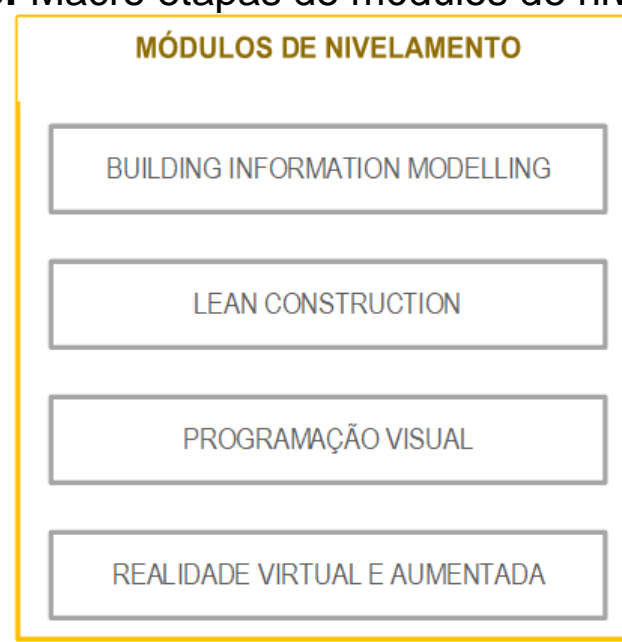

Fonte: Os autores. 
Os módulos serão iniciados no primeiro ano um a cada semestre e em seguida, serão acrescentados os outros dois módulos mantendo os anteriores de acordo com a figura 9.

Figura 9: Fluxo dos módulos no tempo

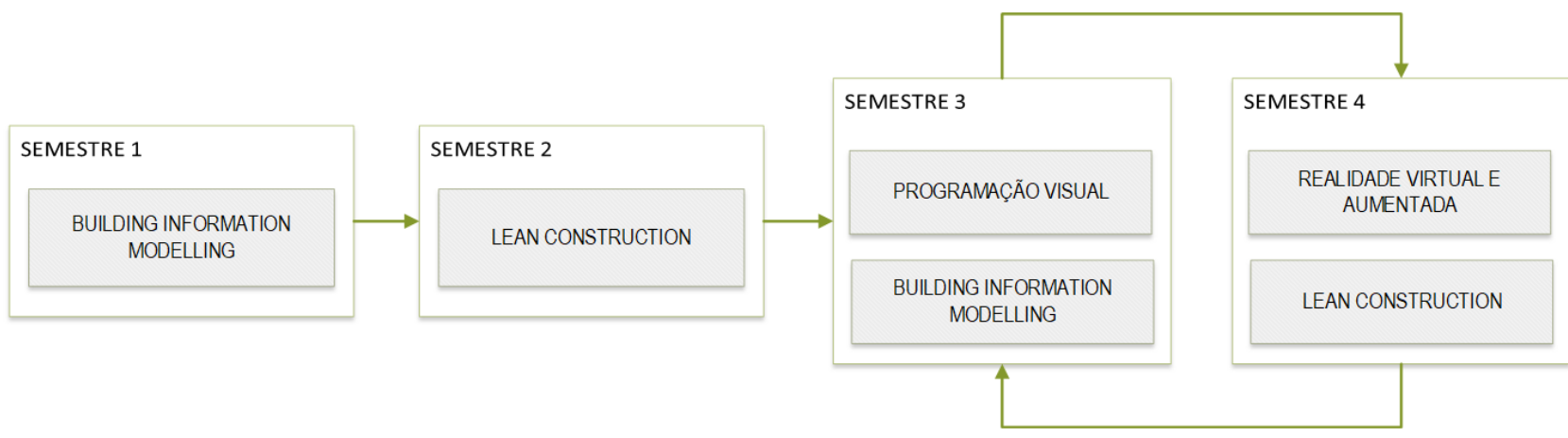

Fonte: Os autores.

\section{CONSIDERAÇÕES FINAIS}

A proposta de nivelamento apresentada neste trabalho, contempla BIM e algumas de suas principais tecnologias associadas com o intuito complementar a formação dos discentes e egressos do curso de engenharia civil frente à crescente demanda do mercado pelo uso do BIM e a necessidade da academia de acompanhar as tecnologias emergentes.

O planejamento do nivelamento foi elaborado com base em uma pesquisa de campo feita com os discentes e egressos que mostrou, dentre alguns aspectos relevantes que estes percebem a importância do BIM para a formação profissional, porém que há uma carência na abordagem do BIM ao longo da graduação, além disso, valorizam abordagens práticas de ensino, de forma que a proposta foi desenvolvida de modo a unir a teoria à prática.

Espera-se com os ciclos de nivelamento proporcionar uma maior integração entre mercado e academia e uma maior disseminação do BIM no curso supracitado. A proposta propõe, ainda, a sanar as lacunas deixadas na formação dos egressos recém saídos do curso.

\section{AGRADECIMENTOS}

Os autores agradecem o apoio do Departamento de Integração Acadêmica e Tecnológica da Universidade Federal do Ceará e do projeto de extensão Escritório de Projetos Integrados de Engenharia.

\section{REFERÊNCIAS}

AZHAR, S. et al. Building information modeling (BIM): now and beyond. Australasian Journal of Construction Economics and Building, 2012, p. 15-28.

BATISTELLO, P.; BALZAN, K. L.; PEREIRA, A. T. C. BIM no ensino das competências em arquitetura e urbanismo: transformação curricular. PARC Pesquisa em Arquitetura 


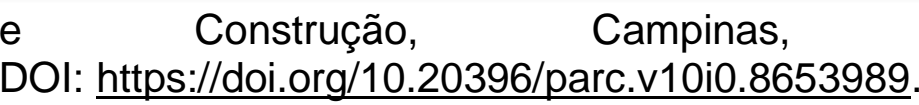

$\mathrm{SP}$,

V.

10, Abr. 2019.

BORGES, R. M. S. et al. Análise da interação entre os usos do BIM e os princípios do Lean Construction em um estudo de caso. In: ENCONTRO NACIONAL DE TECNOLOGIA DO AMBIENTE CONSTRUíDO, 17., 2018, Foz do Iguaçu. Anais. Porto Alegre: ANTAC, 2018.

BRASIL. Presidência da República. DECRETO No 10.306, DE 2 DE ABRIL DE 2020.Disponível em: http://www.in.gov.br/web/dou/-/decreto-n-10.306-de-2-de-abril-de2020-251068946. Acesso em: 09 mai. 2021

BRASIL. Presidência da República. LEI № 14.133, DE 1ํDE ABRIL DE 2021. Disponível em: $\quad$ https://www.in.gov.br/en/web/dou/-/lei-n-14.133-de-1-de-abril-de-2021-311876884. Acesso em: 09 mai. 2021.

CHECCUCCI, Érica de Sousa. Ensino-aprendizagem de BIM nos cursos de graduação em engenharia civil e o papel da expressão gráfica neste contexto. 2014.

COCO JR, Verley Henry; CELANI, Gabriela. Algoritmização do projeto arquitetônico em BIM: uma aplicação na indústria de banheiros pré-fabricados. Gestão \& Tecnologia de Projetos, v. 16, n. 2, p. 73-92, 2021.

CORRÊA, Luciano de Vasconcellos. Contribuições da tecnologia BIM na utilização de estratégias autorregulatórias por estudantes de arquitetura e urbanismo. 2019.

DEBÓN VILLAGRASA, Miquel. La Arquitectura de la información. 2021. Trabalho de Conclusão de Curso. Universitat Politècnica de Catalunya

EASTMAN, C. et al. MANUAL DE BIM. PORTO ALEGRE: BOOKMAN, 2014.

GÓES, Felipe Implantação do BIM utilizando Scrum como abordagem ágil de gerenciamento - um estudo de caso / João Alberto da Costa Ganzo Fernandez Florianópolis, SC, 2019.

GONÇALVES, Sabrina R, et al. Diagnóstico da metodologia BIM entre os anos de 2017 e 2019 no Centro de Tecnologia da UFC. In: XLVIII Congresso Brasileiro de Educação em Engenharia.2020.

KYMMELL, Willem. Building Information Modeling - Planning and Managing

Construction Projects with 4D CDA and Simulations. Ebook. McGraw-Hill, 2008.

LIMA, Weslley Eunathan Fernandes. Proposição de um cenário educacional para ensino do BIM numa perspectiva de ensino híbrido. 2020. Dissertação de Mestrado. Universidade Federal do Rio Grande do Norte.

LIMA, J. O.; RIBEIRO, K. A.. IMPLANTAÇÃO DA CONSTRUÇÃO ENXUTA NA INDÚSTRIA DA CONSTRUÇÃO CIVIL. Revista Gestão Empresarial - Rge, Três Lagoas, v. 6, n. 2, p. 64-81, 16 dez. 2020. Disponível em: https://trilhasdahistoria.ufms.br/index.php/disclo /article/view/12161. Acesso em: 09 maio 2021.

LUCENA, Arthur Felipe Echs. Desenvolvimento de recursos baseados em jogos e realidade virtual para capacitação em segurança do trabalho de gestores da 
construção civil. 2019. 174f. Dissertação (Mestrado) - Programa de Pós-graduação em Engenharia Civil - Universidade Estadual de Londrina, Londrina, 2019.

PEREIRA, Igor Patricio. Realidade Virtual Aplicada na Engenharia. Engenharia CivilTubarão, 2020.

PEREIRA, Pedro Augusto Izidoro; RIBEIRO, Rochele Amorim. A Inserção de BIM no curso de graduação em Engenharia Civil. International Journal on Alive Engineering Education, v. 2, n. 2, p. 17-30, 2015.

MINISTÉRIO DA EDUCAÇÃO. Diretrizes curriculares: Cursos de graduação. Disponível em: http://portal.mec.gov.br/component/content/article?id=12991. Acesso em: 22 abr. 2021.

SILVA, Clara Beatriz Fidelis da et al. Realidade Virtual como ferramenta facilitadora no planejamento de canteiro de obras. In: CONGRESSO NACIONAL DE CONSTRUÇÃO DE EDIFÍcIOS.

\title{
BIM LEVELING PROPOSAL FOR DISCENT AND EGRESSES FROM THE CIVIL ENGINEERING COURSE OF THE FEDERAL UNIVERSITY OF CEARÁ
}

\begin{abstract}
In the sphere of the Civil Engineering course at the Federal University of Ceará, there is a lack regarding the applications of Building Information Modeling BIM. In view of this, complementary actions were necessary to permeate the undergraduate course, such as the creation of an optional discipline, holding events, as well as the creation of an extension project. In this scenario, until then the actions developed in a more intensive way were directed to the students of the course, as is the case of the optional discipline and the extension project mentioned above. The National Curricular Guidelines for the civil engineering course recommends the development of activities that bring students closer to the professional environment. In this context, we have the BIM BR strategy which, among its objectives, encourages training in BIM, among others. Thus, the objective of this work is to propose a leveling of BIM, associated with complementary tools and technologies, to students and graduates of the Civil Engineering course, with the aim of promoting BIM integrated between academia and the market in a more incisive and directed. In this scenario, four training modules will be developed under themes: BIM, Lean, Visual programming, virtual and augmented reality. These modules will be conducted in a collaborative manner between students, graduates and invited professionals, in order to promote the engagement of those involved. It is expected that at the end of the process, participants will develop both experience reports so that other universities can replicate the action, as well as, develop new solutions regarding the engineering of what has been experienced.
\end{abstract}

Keywords: BIM, Civil Engineering, UFC, leveling. 